\title{
Diacronie
}

Studi di Storia Contemporanea

$\mathrm{N}^{\circ} 28,4 \mid 2016$

La voce del silenzio

\section{Tra spionaggio e difesa: il Westarbeit tedesco- orientale nella fase di détente}

\section{Federica Addis}

\section{(QpenEdition \\ Journals}

\section{Edizione digitale}

URL: http://journals.openedition.org/diacronie/4811

DOI: $10.4000 /$ diacronie.4811

ISSN: 2038-0925

\section{Editore}

Association culturelle Diacronie

\section{Notizia bibliografica digitale}

Federica Addis, «Tra spionaggio e difesa: il Westarbeit tedesco-orientale nella fase di détente »,

Diacronie [Online], № 28, 4 | 2016, documento 13, Messo online il 29 décembre 2016, consultato il 30 avril 2019. URL : http://journals.openedition.org/diacronie/4811; DOI : 10.4000/diacronie.4811 


\title{
Diacronie
}

N. 28 | 4|2016 La voce del silenzio: intelligence, spionaggio e conflitto nel XX secolo

\section{3/}

\section{Tra spionaggio e difesa: il Westarbeit tedesco- orientale nella fase di détente}

\author{
Federica ADDIS *
}

Il contributo intende rendere conto del lavoro di spionaggio e di controspionaggio messo in atto dal Ministero per la Sicurezza Statale della Repubblica Democratica Tedesca (meglio conosciuto come Stasi) durante il periodo di détente. L'apertura verso occidente comportò per il Ministero una lunga serie di compiti nuovi e gravosi, per assolvere ai quali il suo apparato subì un'espansione eccezionale ed impressionante. Facendo perno sulla storiografia tedesca prodotta sul tema, l'articolo ha voluto illustrare gli sviluppi del Ministero, le principali azioni compiute e, più in generale, la sua complessa reazione, fatta di spionaggio e di difesa, di fronte a questi importanti rivolgimenti politici.

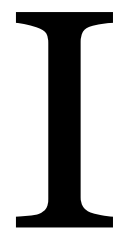

1 Ministero per la Sicurezza Statale della Repubblica Democratica Tedesca (meglio conosciuto come Stasi) disponeva di un organo, l'Hauptverwaltung Aufklärung (HVA), dedito allo spionaggio verso l'estero. Il dipartimento HVA fu circondato, già nel corso della sua esistenza, da un'aurea leggendaria, di cui godette anche a causa della misteriosa figura di Markus Wolf, «l'uomo senza volto» ${ }^{1}$ che ne guidò la direzione tra il 1956 ed il 1986. Se, anche con il contributo dello stesso Wolf, le pubblicazioni autobiografiche e le memorie di ex-dipendenti dell'HVA si sono moltiplicate già all’indomani del crollo del Muro, la ricerca storica ha trovato sul proprio percorso qualche ostacolo in più. Oltre ad

\footnotetext{
${ }^{1}$ La più importante tra le sue autobiografie fu tradotta in diversi paesi. Cfr. la versione italiana: WOLF, Markus, L'uomo senza volto, Milano, Rizzoli, 1997.
} 
una limitata esperienza della storiografia tedesca nelle analisi sui servizi segreti ${ }^{2}$, gli studiosi d'oltralpe si sono dovuti accontentare di una base documentaria ridotta e frammentaria. Ancor prima della Wende, probabilmente intuendo i rivolgimenti radicali che si sarebbero verificati di lì a poco, infatti, i collaboratori dell'HVA procedettero alla distruzione di numerosi incartamenti conservati nei suoi archivi: la maggior parte della documentazione ad esso relativa è così andata perduta3 .

Il lavoro di spionaggio non era però appannaggio esclusivo dell'HVA: quest'ultimo non era un’istituzione a sé stante, ma era integrato nella struttura del Ministero per la Sicurezza. Le modalità operative della Stasi si caratterizzavano per una stretta interdipendenza tra spionaggio (Aufklärung) e attività di difesa (Abwehr)4; un assetto che imponeva una costante collaborazione tra l'HVA e le altre sezioni dell'organo di sicurezza. Il lavoro era in questo senso ampiamente distribuito: sul piano orizzontale, vari dipartimenti comprendevano nella loro attività anche incarichi di intelligence, mentre a livello verticale, ciascun distaccamento locale del Ministero disponeva di una sezione XV5, impegnata in funzioni di Aufklärung. Difesa e spionaggio erano quindi meccanismi parziali di un sistema integrato, esattamente come accadeva nel KGB sovietico, assunto a modello di riferimento ${ }^{6}$. Proprio alla luce di questa organizzazione, all'interno del Ministero stesso non ci si riferiva tanto al "semplice" spionaggio, quanto piuttosto ad un più generale «Arbeit im und nach dem Operationsgebiet», letteralmente traducibile come «lavoro nel e verso l'ambito operativo». Il concetto di Operationsgebiet mirava ad identificare un determinato "ambito nemico", che diveniva obiettivo di azioni perpetrate con mezzi e metodi tipici dei servizi segreti: nonostante esso non fosse quindi da ridurre ad una mera collocazione territoriale, ma stesse ad indicare qualsiasi ambito in cui si fosse trovato il nemico, l'Operationsgebiet a cui la DDR indirizzava molta della propria attività aveva dei confini ben definiti, quelli della vicina Repubblica Federale Tedesca (RFT) e di Berlino Ovest7.

2 MÜLLER-ENBERGS, Helmut, Die Erforschung der Westarbeit des MfS. Stand und Perspektiven, in SUCKUT, Siegfried, WEBER, Jürgen, (herausgegeben von) Stasi-Akten zwischen Politik und Zeitgeschichte. Eine Zwischenbilanz, München, Olzog, 2003, p. 265.

${ }^{3}$ BOOß, Christian, «"Arbeit im und nach dem Operationsgebiet", Die Unterlagen zur Westarbeit des MfS in den Beständen der BStU», in Deutschland Archiv, 43, 6/2010, pp. 1078-1080.

${ }^{4}$ KNABE, Hubertus (herausgegeben von), Die West-Arbeit des MfS. Zusammenspiel von „Aufklärung und Abwehr“, Berlin, Ch. Links, 1999; FRICKE, Karl Wilhelm, «Ordinäre Abwehr Elitäre Aufklärung? Zur Rolle der Hauptverwaltung A im Ministerium für Staatssicherheit», in Aus Politik und Zeitgeschichte, 50, 1997, pp. 17-26.

5 KNABE, Hubertus, «Die Stasi als Problem des Westens. Zur Tätigkeit des MfS im "Operationsgebiet“», in Aus Politik und Zeitgeschichte, 50, 1997, pp. 3-16, p. 8.

"SIEBENMORGEN, Peter, „Staatssicherheit" der DDR. Der Westen im Fadenkreuz der Stasi, Bonn, Bouvier Verlag, 1993, pp. 99, 103.

${ }^{7}$ BOOß, Christian, op. cit., p. 1077. 
Per riferirsi al lavoro della Stasi "nell'ambito operativo", sia la pubblicistica che la storiografia utilizzano comunemente il vocabolo composto Westarbeit: la locuzione, sebbene non strettamente scientifica e mai utilizzata all'interno del Ministero, è efficace nel rendere con immediatezza l'idea di un'attività complessa, proprio perché poggiata su di un'interdipendenza tra spionaggio e difesa, oltre che sulla collaborazione di svariati dipartimenti per la sua realizzazione ${ }^{8}$. Proprio in conseguenza di questa sua particolare organizzazione "diffusa" e "composita", il Westarbeit della Stasi non è documentato esclusivamente negli atti dell'HVA. La ricerca storiografica ha quindi potuto integrare i documenti sopravvissuti alla distruzione degli archivi di questo dipartimento con lo studio degli incartamenti conservati in altre sezioni del Ministero e con i database Rosenholz e SIRA, registri elettronici scoperti successivamente che, nonostante diverse problematicità legate alla loro natura e alla storia del loro ritrovamento, sono risultati di fondamentale importanza per la delineazione di un quadro d'insieme9. Si sono in questo modo potuti ricostruire la struttura, l'organizzazione, le funzioni e le caratteristiche del lavoro verso occidente del Ministero in generale, e dell'HVA in particolare, producendo studi di riferimento sul tema, soprattutto tra la fine degli anni Novanta e la prima metà dei Duemila ${ }^{10}$.

Sebbene quindi si sia potuto compensare, almeno in parte, alle lacune documentarie del dipartimento HVA, e nonostante la presenza di importanti pubblicazioni generali e di numerosi studiosi che, anche grazie al significativo appoggio di alcune fondazioni per l'elaborazione della storia della Germania divisa ${ }^{11}$, abbiano rivolto la propria attenzione al Ministero per la Sicurezza dopo il 1990, la vastità del tema e le problematicità tipiche di un ambito di studio così delicato, lasciano spazio a numerose integrazioni ed approfondimenti. Appare a questo proposito singolare come

\footnotetext{
${ }^{8}$ Per i concetti di Arbeit im und nach dem Operationsgebiet e di Westarbeit, Cfr. Ibidem, pp. $1077,1078$.

${ }^{9}$ Per una trattazione dei database e del loro utilizzo, cfr. MÜLLER-ENBERGS, Helmut, "Rosenholz": Probleme mit der Kartei der DDR-Spionage, in TIMMERMANN, Heiner (herausgegeben von), Die DDR in Europa - Zwischen Isolation und Öffnung, Münster, LIT, 2005, pp. 174-189; HECHT, Jochen, "Rosenholz“ und SIRA - archivalische Quellen zur Geschichte der Hauptverwaltung Aufklärung ( $H V A$ ) des MfS, in UNVERHAU Dagmar (herausgegeben von), Hatte „Janus“ eine Chance?: Das Ende der DDR und die Sicherung einer Zukunft der Vergangenheit, Münster, LIT, 2003, pp. 99-112.

${ }_{10}$ Cfr. la bibliografia aggiornata sul sito del Der Bundesbeaufragte der Unterlagen des Staatssicherheitsdienstes der ehemaligen Deutschen Demokratischen Republik (BStU), URL:

<https://www.bstu.bund.de/SharedDocs/Downloads/DE/Publikationsverzeichnis_des_BStU.p df? _blob=publicationFile $>$ [consultato il 28 settembre 2016].

Per quanto concerne invece la documentazione disponibile per la ricerca sul Westarabeit, cfr. GIESEKE, Jens, "East German Espionage in the Era of Détente», in Journal of Strategic Studies, 31, 3/2008, pp. 397-398.

${ }^{11}$ Per la Stasi l'istituzione di riferimento è senza dubbio il BStU, il quale ha patrocinato, fin dai primi anni Novanta, moltissime ricerche.
} 
non esista, ancora, un'analisi strutturata e specificatamente dedicata alla gestione ed agli effetti del Westarbeit tedesco-orientale in quella particolare fase di distensione che, tra la fine degli anni Sessanta e nel corso dei Settanta, cambiò in modo sostanziale le condizioni della Guerra Fredda. Alcune analisi, certo, esistono: esse, senza dubbio significative e rilevanti, sono però parte integrante di studi più generali sulla storia del Ministero o di un suo dipartimento, oppure si tratta di articoli o contributi in volumi collettanei, perlopiù volti ad indagare aspetti particolari o vicende specifiche. Questa carenza risulta ancor più sorprendente se si tiene in considerazione come la détente rappresentò un momento di svolta per la vita politica ed istituzionale della DDR: il Trattato Fondamentale con la vicina Germania Occidentale nel 1972, così come gli accordi bilaterali conclusi con numerosi altri stati in quei mesi, nonché la firma dell'Atto Finale di Helsinki nel 1975, segnarono la fine dell'isolamento della Germania comunista, il suo riconoscimento da parte di ben 109 Paesi nel giro di pochi anni e la sua entrata nell'ONU, sancendone la definitiva legittimazione nell'arena politica internazionale.

Un'analisi comprensiva di tale variegata e disorganica letteratura sul tema, permette comunque di fornire un quadro indicativo dell'attività del Ministero per la Sicurezza durante questa particolare congiuntura storica. Il primo dato ad emergere con chiarezza è il tratto peculiare dell'approccio con cui la Stasi si rivolse, fin dai primi sviluppi, alla distensione. Esso si concretizzò in un atteggiamento ambivalente: se da una parte il Ministero agì, in quanto fedele sostenitore del partito e delle sue decisioni, per una risoluzione positiva della politica di avvicinamento all'occidente, dall'altra la sua attenzione rimase costantemente focalizzata sul vaglio delle conseguenze che il processo di distensione avrebbe potuto portare con sé ${ }^{12}$, sia in termini di opportunità, ma soprattutto in termini di potenziali pericoli per la stabilità del paese.

La Stasi si occupò della détente fin dalle primissime battute, riuscendo ad esempio, come dimostrano una serie di documenti sopravvissuti alla distruzione, a tenere aperto un canale secondario sulle negoziazioni. Alcuni membri della delegazione tedescoorientale riportavano lo sviluppo dei colloqui non solo ai propri responsabili politici, ma anche alla Stasi ${ }^{13}$, tenendo con il Ministero un contatto diretto. Secondo la studiosa statunitense Mary Elise Sarotte, più precisamente, ben quattro dei nove componenti principali della delegazione tedesco-orientale nella fase di trattativa (1969-1974) sarebbero stati, a diverso titolo, collaboratori del Ministero per la Sicurezza: due di essi

\footnotetext{
${ }^{12}$ GIESEKE, Jens, Die hauptamtlichen Mitarbeiter der Staatssicherheit. Personalstruktur und Lebenswelt 1950-1989, Berlin, Ch. Links, 2000, p. 304.

${ }^{13}$ GIESEKE, Jens, «East German Espionage in the Era of Détente», cit., pp. 419-420.
} 
(Alwin Brandt e Helmut Nacke) sarebbero stati attivi come hauptamtlichen Mitarbeiter ${ }^{14}$, mentre altri due (Karl Seidel e Heinz Gerber) avrebbero agito nella veste di inoffiziellen Mitarbeiter ${ }^{15}$. Il principale rappresentante della DDR, il Segretario di Stato Michael Kohl, inoltre, inviava regolarmente rapporti dettagliati sui propri colloqui con Egon Bahr: proprio la congruenza delle relazioni di Kohl con alcune informative direttamente inviate al Ministro per la Sicurezza Erich Mielke da un IM con nome in codice "Koran", sarebbe indice del fatto che il Segretario di Stato informasse il Ministero anche per via «non ufficiale» ${ }^{16}$. Vari documenti testimoniano, poi, come la raccolta di informazioni passasse anche per l'intercettazione ambientale dei colloqui, attraverso cui la Stasi controllava non soltanto le posizioni nemiche, ma anche il comportamento dei propri emissari ${ }^{17}$. Vi erano, infine, canali esterni alla delegazione ufficiale, come ad esempio quello costituito da Hermann von Berg, un membro "critico" del SED che svolse un importante ruolo di trait d'union tra i capi di governo Brandt e Stoph: sulle trattative aggiornava costantemente la Stasi, di cui era collaboratore non ufficiale con nome in codice "Günther"18.

L'attività del Ministero in questa fase negoziale non si limitò alla raccolta di informazioni, concretizzandosi piuttosto in vere e proprie aktive Maßnahmen, "manovre attive" nei confronti del vicino occidentale. Con lo scopo di rafforzare le posizioni tedesco-orientali nella RFT e per favorire, in questo modo, la conclusione positiva delle trattative, il Ministero si impegnò ad appoggiare quelle che venivano identificate come «forze progressiste» all'interno del panorama politico della Germania Occidentale: già per le elezioni federali del 1965, ad esempio, il Ministro per la Sicurezza Mielke dispose di «mobilitare tutte le forze contro il governo Erhard e la sua

\footnotetext{
${ }^{14}$ Con hauptamtlichen Mitarbeiter, HM, si identificavano i lavoratori dipendenti presso il Ministero per la Sicurezza Statale.

${ }^{15}$ SAROTTE, Mary Elise, Spying Not Only On Strangers: Documenting Stasi Involvement In Cold War German-German Negotiations, in BOGLE, Lori Lyn, (edited by) The Cold War. Cold War Espionage And Spying, vol. IV, New York-London, Routledge, 2001, pp. 75-76.

Gli inoffiziellen Mitarbeiter, IM, collaboravano con il Ministero per la Sicurezza «in modo coperto», ma non ne erano dipendenti fissi. Erano il «collegamento segreto tra la sicurezza e la società»: i loro compiti andavano dalla raccolta di informazioni all'esercizio di influenze sulle persone fino all'offerta di aiuto logistico [Cfr. MÜLLER-ENBERGS, Helmut, Die inoffiziellen Mitarbeiter, Berlin, BStU, 2008, p. 5].

${ }^{16}$ SAROTTE, Mary Elise, Spying Not Only On Strangers, cit., pp. 76-77; GIESEKE, Jens, «East German Espionage in the Era of Détente», cit., p. 420.

${ }^{17}$ SAROTTE, Mary Elise, Spying Not Only On Strangers, cit., pp. 73-75; BANGE, Oliver, Zwischen Bedrohungsperzeption und sozialistischen Selbstverständnis. Die DDRStaatssicherheit und westliche Transformationsstrategien 1966-1975, in DIEDRICH, Torsten, SÜB, Walter (a cura di), Militär und Staatssicherheit im Sicherheitskonzept der WarschauerPakt-Staaten, Berlino, Ch. Links, 2010, p. 274.

${ }^{18}$ KNABE, Hubertus, Die unterwanderte Republik. Stasi im Westen, Berlino, Propyläen, 1999, pp. 31-38.
} 
politica revanscista», mentre erano da evitare «manovre contro l'SPD»19. Un'attività che proseguì negli anni successivi: così, ad esempio, in una consultazione degli organi di sicurezza a Budapest nel dicembre 1970 (quando, quindi, Brandt era Cancelliere da più di un anno), la delegazione tedesco-orientale esprimeva il suo impegno per «sostenere le forze, sia in RFT che al di fuori dei suoi confini, che valutino la situazione in modo realistico e che si preoccupino realmente per la pace in Europa, contro le forze revansciste e militari» ${ }^{20}$, mentre, analogamente, per le elezioni federali del 1976 «il compito consiste[va] nella lotta contro le forze più reazionarie, apertamente revansciste, nemiche della distensione e conservatrici, in particolare nella CDU/CSU, e contro i circoli operanti dietro ad esse ${ }^{21}$, anche per mezzo di un approfondimento delle rivalità e dei contrasti nello schieramento imperialista ${ }^{22}$. Proprio la CDU/CSU era considerata la forza conservatrice per eccellenza, oltre che il maggior avversario politico dell'SPD. Verso di essa vennero dunque rivolte diverse manovre, alla cui base stava un antifascismo propagandistico, che mirava a screditare pubblicamente esponenti del partito, accusandoli di connivenze o collaborazioni con il regime hitleriano. Una strategia che conobbe il proprio culmine nella campagna contro il Presidente Federale (cristiano-democratico, appunto), Heinriche Lübke: le accuse tedesco-orientali di corresponsabilità nella costruzione e nel funzionamento dei campi di concentramento lo costrinsero alle dimissioni nel $1968^{23}$.

Pensare che il Ministero per la Sicurezza si stesse impegnando in un'autentica politica di appoggio all'SPD, in una convinta attività in favore di una politica di distensione, o, come lasciano intendere alcune memorie di ex-collaboratori della Stasi, in una politica di pace per l'Europa ${ }^{24}$, è però decisamente fuorviante. Innanzitutto, il corso «socialdemocratico» ed «anticomunista» dell'SPD era comunque osservato con diffidenza $^{25}$. Apparivano inoltre chiari i possibili effetti negativi di un'apertura ad occidente: era d'altronde piuttosto palese come tutti gli accordi al vaglio delle intense trattative di quei mesi (dall'Accordo di Transito del 1971, al Trattato sul Traffico del 1972, fino ad arrivare al Trattato Fondamentale del 21 Dicembre 1972), avrebbero

\footnotetext{
${ }^{19}$ Ibidem, p. 30.

${ }^{20}$ Ibidem, p. 38.

${ }^{21}$ Ibidem, p. 40.

${ }^{22}$ Ibidem.

${ }^{23}$ Ibidem, pp. 121-152.

${ }^{24}$ Cfr. ad esempio: GROßMANN, Werner, Aufgaben und Ergebnisse der Aufklärung der DDR zur Ausschaltung des Überraschungsmomentes, in EICHNER, Klaus, SCHRAMM, Gotthold (herausgegeben von), Spionage für den Frieden. Eine Konferenz in Berlin am 7. Mai 2004, Berlin, edition ost, 2004, pp. 76-84; SCHWANITZ, Wolfgang, Sicherung der DDR als Beitrag zur Sicherung des Friedens in Europa, in EICHNER, Klaus, SCHRAMM, Gotthold (herausgegeben von), op. cit., 2004, 85-91.

${ }^{25}$ BANGE, Oliver, op. cit., pp. 266-272.
} 
comportato gravose incombenze per il Ministero ${ }^{26}$. Secondo i timori, mai celati, di Mielke, il contatto con l'esterno avrebbe potuto destabilizzare la situazione politica del paese: il Ministro leggeva negli avvenimenti in corso, nella politica di contatto e negli intenti della Ostpolitik «il rafforzato tentativo da parte del nemico di intensificare la politica di diversione politico-ideologica e di indebolimento» ${ }^{27}$. Proprio il concetto di politisch-ideologische Diversion (nei documenti della Stasi abbreviata in PID), costituiva il punto cardine di questa percezione: assumendo il carattere di una vera e propria «dottrina della sicurezza» ${ }^{28}$, questo quadro di riferimento venne a più riprese utilizzato per fornire una spiegazione alle crisi interne al paese ${ }^{29}$. Il PID era una vera e propria strategia di lotta, messa in atto dall'Occidente:

Con la diversione politico-ideologica il nemico aspira ad obiettivi sovversivi in un processo a lungo termine e a più stadi. Essi consistono in una disgregazione della consapevolezza socialista o nel turbamento ed impedimento della sua formazione, nel logoramento della fiducia di ampi settori di popolazione nei confronti della politica dei partiti comunisti e dei paesi socialisti, nell'ispirazione di comportamenti antisocialisti fino alla perpetrazione di crimini contro lo stato, nella mobilitazione di forze nemiche e negative nei paesi socialisti, nello sviluppo di una base nemica, ideologica e personale, per ispirare attività clandestine, così come nel provocare insoddisfazione, inquietudine, passività e insicurezza politica tra ampi strati di popolazione $e^{30}$.

La firma dei trattati forniva un terreno fertile per la messa in atto di questa strategia: la nuova presenza di corrispondenti e diplomatici, l'intensificazione dei rapporti economici, politici e personali, così come l'aumento dei viaggi transfrontalieri, infatti, mettevano a disposizione una lunga serie di possibilità concrete di minaccia ${ }^{31}$. L'attività del nemico disponeva in questo senso di mezzi e metodi non soltanto nuovi, ma particolarmente pericolosi perché «più raffinati»32: per questa ragione il Ministro si

${ }^{26}$ GIESEKE, Jens, Die hauptamtlichen Mitarbeiter der Staatssicherheit. Personalstruktur und Lebenswelt 1950-1989, cit., p. 306.

${ }^{27}$ KNABE, Hubertus, «Die Stasi als Problem des Westens. Zur Tätigkeit des MfS im "Operationsgebiet"», cit., p. 6. Corsivo nell'originale.

${ }^{28}$ GIESEKE, Jens, Die hauptamtlichen Mitarbeiter der Staatssicherheit. Personalstruktur und Lebenswelt 1950-1989, cit., p. 312.

${ }^{29}$ Anche per gli eventi del 17 Giugno 1953, ad esempio, si pensò a dei possibili mandanti in occidente. KNABE, Hubertus, «Die Stasi als Problem des Westens. Zur Tätigkeit des MfS im „Operationsgebiet“", cit., p. 4.

${ }^{30}$ SUCKUT, Siegfried (herausgegeben von), Das Wörterbuch der Staatssicherheit. Definitionen zur „politischen-operativen Arbeit“, Berlin, Ch. Links, 1996, pp. 303, 304.

${ }^{31}$ KNABE, Hubertus (herausgegeben von), West-Arbeit des MfS, cit., p. 92.

${ }^{32}$ LABRENZ-WEIß, Hanna, Die Hauptabteilung II: Spionageabwehr, Berlin, BStU, 1998, p. 49. 
preoccupò di sottolineare la necessità di indirizzare «grandi sforzi per l'innalzamento dell'efficienza del nostro lavoro», con lo scopo di «contrastare, preventivamente ed in modo offensivo, tutti i tentativi del nemico di turbare ed intralciare l'organizzazione del sistema sociale sviluppato del socialismo, sia con attacchi dall'esterno che all'interno della DDR»33. Occorreva in particolare saper chiarire per tempo «piani, intenzioni, manovre, mezzi e metodi dei centri nemici e gli altri punti di partenza dell'attività sovversiva»34. Ancora Mielke, ad una conferenza dei dirigenti del Ministero alla vigilia della firma del Trattato Fondamentale, affermò che le negoziazioni erano state una «lotta di classe ancora più dura», per poi spiegarsi meglio: «con questo intendo rendere evidente che la lotta continua e che noi, come Ministero per la Sicurezza, dobbiamo essere totalmente preparati a combatterla» 35 .

Questi timori avevano d'altronde già trovato un'esemplare concretizzazione ancor prima della firma dei trattati. Durante le negoziazioni, infatti, furono organizzati due incontri tra i massimi esponenti dei due governi, uno ad Erfurt, nella DDR, il secondo a Kassel, nella BRD: il carattere competitivo dell'atteggiamento tedesco-orientale si rese evidente già nella scelta dei nomi in codice assegnati alle misure di sicurezza per i due vertici, rispettivamente Konfrontation I e Konfrontation II. Il primo dei due incontri, quello in Germania Orientale, venne fissato per il 19 Marzo 1972 e segnò una vera e propria debacle per la Stasi, secondo natura responsabile per la messa in sicurezza dei colloqui. Le misure previste dal Ministero non furono sufficienti a contenere la forte reazione delle circa $\mathbf{1 . 5 0 0}$ persone riunite in piazza per l'arrivo del Cancelliere Willy Brandt: quest'ultimo fu accolto con dimostrazioni di calore inaspettate, che misero in seria difficoltà le forze della Stasi dispiegate per l'occasione36. L'analisi critica, tempestivamente effettuata dal Segretariato del Comitato Centrale, si inseriva nella cornice interpretativa di cui sopra: «La preparazione dell'incontro di Erfurt non è stato completamente riconosciuta come una componente-chiave nel conflitto di classe tra socialismo ed imperialismo. La pericolosità del nemico di classe e il suo intento volto a preparare e condurre un atto organizzato e provocatorio non è stato riconosciuto»37. Una parte degli studiosi tende a considerare gli avvenimenti di Erfurt come un reale imprevisto per il Ministero; un'altra parte, invece, sostiene che un simile

\footnotetext{
${ }^{33}$ KNABE, Hubertus (herausgegeben von), West-Arbeit des MfS, cit., p. 92.

${ }^{34}$ Ibidem.

${ }^{35}$ GIESEKE, Jens, «Auf dem Wege zu einer Gesellschaftsgeschichte der Repression in der DDR», in: Erinnern! Rundbrief der Stiftung Gedenkstätten Sachsen-Anhalt, 1/2012, pp. 27-41, p. 31.

${ }^{36}$ Ibidem, p. 29. Per una narrazione più ampia degli avvenimenti di quel giorno, cfr. SAROTTE, Mary Elise, Dealing with the devil. East Germany, Détente, and Ostpolitik, Chapel Hill, The University of North Carolina Press, 2001, pp. 46-48.

${ }^{37}$ SAROTTE, Mary Elise, Dealing with the Devil, cit., p. 46. Corsivo nell'originale.
} 
sviluppo sia stato volutamente consentito dalla Stasi, proprio per rendere evidente le conseguenze che un'apertura ad Occidente avrebbe portato con sé38.

Preoccupazioni analoghe accompagnarono il momento culminante del processo di distensione, raggiunto con la firma dell'Atto Finale di Helsinki nel 1975. Anche in questo caso, il Ministero per la Sicurezza non nascondeva i propri timori, che Mielke esprimeva con il solito vocabolario:

Attraverso la diversione politico-ideologica ed una politica di contatto/attività di contatto come elementi di guerra psicologica, l'avversario tenta in misura crescente di esercitare influenza sui cittadini della DDR, di manipolarli verso un'ideologia borghese e di destare in loro la decisione di abbandonare la $\mathrm{DDR}^{39}$.

Già l'anno precedente il Ministro aveva avvertito che il processo in corso sarebbe servito al nemico occidentale per raggiungere alcuni scopi specifici, quali un' «ampia infiltrazione ideologica», la realizzazione di una «dipendenza economica» e l'esercizio di pressioni sui paesi socialisti attraverso la "parola d'ordine "libera circolazione" $\gg 40$.

Tornava la solita ambivalenza: il Ministero per la Sicurezza si trovò incastrato tra il significato pubblico-politico dell'accordo e la complicata gestione dei suoi lasciti. La firma del Trattato, a livello politico, fu infatti celebrata come un vero e proprio successo: sancendo principi quali il rispetto della sovranità, l'integrità territoriale o il non intervento negli affari interni degli altri paesi, l'Atto Finale sembrava confermare l'affermazione tedesco-orientale nel quadro politico europeo ed internazionale, oltre che il successo della sua politica estera. L'evento meritò, per questa ragione, un'adeguata risonanza mediatica: l'Atto fu quindi proposto nella sua interezza sulla prima pagina del «Neues Deutschland». La scelta si rivelò però poco accorta da un punto di vista di stabilità interna: immediatamente dopo la firma del Trattato, il Ministero per la Sicurezza dovette registrare una vera e propria esplosione del numero di richieste di espatrio, che per la maggior parte si appellavano ai diritti sanciti dal "cesto III" pattuito ad Helsinki ${ }^{41}$. I problemi che venivano così emergendo, richiedevano un adeguato adattamento organizzativo, a cui la Stasi provvide con alacre efficienza: nel dicembre 1975 entrò in funzione il Zentraler Koordinierungsgruppe

${ }^{38}$ GIESEKE, Jens, «Auf dem Wege zu einer Gesellschaftsgeschichte der Repression in der DDR», cit., p. 30.

${ }^{39}$ EISENFELD, Bernd, «Reaktionen der DDR-Staatssicherheit auf Korb III des KSZE-Prozess», in Deutschland Archiv, 6/2005,

40 HANISCH, Anja, Die DDR im KSZE-Prozess 1972-1985. Zwischen Ostabhängigkeit, Westabgrenzung und Ausereisebewegung, München, Oldenbourg, 2012, p. 109.

${ }^{41}$ EISENFELD, Bernd, op. cit., p. 1001; GIESEKE, Die hauptamtlichen Mitarbeiter der Staatssicherheit. Personalstruktur und Lebenswelt 1950-1989, cit., pp. 309, 310. 
(ZKG), con il compito di impedire la fuga e gli aiuti volti a permetterla, e di gestire le sempre più numerose domande di espatrio42. A pochi mesi dalla firma del trattato, nell'ottobre 1976, Mielke ordinò il respingimento di tutte quelle richieste di trasferimento verso la Repubblica Federale e Berlino Ovest che si fossero appellate al trattato del 1975 o che avessero reclamato un'inosservanza, da parte dello Stato, di obblighi derivanti dal diritto internazionale o dai regolamenti interni 43 . Nel marzo del 1977 Mielke espose poi alcune esigenze operative: si doveva procedere a combattere gli «ispiratori occidentali» delle istanze di espatrio (in particolare le diverse organizzazioni di aiuto logistico e supporto politico); dovevano inoltre essere svolte accurate verifiche su tutti i richiedenti; occorreva, infine, rintracciare quelle persone che avrebbero potuto presentare una richiesta d'espatrio, in modo da poter procedere precauzionalmente alla loro «lavorazione»44. Era necessario cioè che il Ministero si impegnasse a riconoscere preventivamente i potenziali effetti negativi dell'apertura ad occidente, in modo da poter intraprendere le adeguate manovre: venne così, per la prima volta in modo preciso, delineata una nuova strategia per il mantenimento della sicurezza, con la quale «la presenza nella società da parte del Ministero raggiunse una nuova qualità»45. La gestione dell'ordine interno, però, doveva ormai tenere conto della nuova posizione assunta dal Paese a livello internazionale, la quale imponeva il mantenimento di una rispettabilità a livello pubblico: erano così da evitare manovre apertamente repressive, in favore di procedimenti meno appariscenti. A partire dal gennaio 1976 cominciò ad essere stabilmente utilizzata la cosiddetta Zersetzung, letteralmente "decomposizione": era una pratica che mirava alla demoralizzazione ed alla paralisi di gruppi o di singole persone che svolgevano attività di opposizione al regime ${ }^{46}$ (tra queste anche i possibili richiedenti espatrio), attraverso manovre di discredito pubblico, organizzazione di insuccessi lavorativi o sociali, sfruttamento o creazione di contrapposizioni e diffidenze all'interno dei gruppi, determinazione di sentimenti di sfiducia47. Di nuovo, l'occasione fu sfruttata anche per l'attività di raccolta di notizie, apparentemente particolarmente produttiva in questa fase: dal database SIRA, che registrava le informazioni in entrata dal dipartimento per lo spionaggio, si può infatti notare come siano state inserite ben

${ }^{42}$ SÜB, Walter, Die KSZE-Prozess der 1970er Jahre aus der Perspektive der DDRStaatssicherheit, in DIEDRICH, Torsten, SÜß, Walter (herausgegeben von), Militär und Staatssicherheit im Sicherheitskonzept der Warschauer-Pakt-Staaten, cit., p. 332.

${ }^{43}$ EISENFELD, Bernd, op. cit., p. 1001.

${ }^{44}$ GIESEKE, Jens, Die hauptamtlichen Mitarbeiter der Staatssicherheit. Personalstruktur und Lebenswelt 1950-1989, cit., p. 311.

${ }^{45}$ Ibidem.

${ }^{46}$ SÜß, Walter, op. cit., p. 329.

${ }^{47}$ GIESEKE, Jens, Die hauptamtlichen Mitarbeiter der Staatssicherheit. Personalstruktur und Lebenswelt 1950-1989, cit., p. 313. 
3.682 voci con rimando, diretto od indiretto, alla Conferenza per la Sicurezza e la Cooperazione in Europa ${ }^{48}$.

Questa tormentata attenzione per tutto il corso della distensione ebbe considerevoli effetti a livello fattuale: essa contribuì, in maniera decisiva, all'incredibile espansione che il Ministero per la Sicurezza conobbe a partire dal 1968. Naturalmente, altri eventi concorsero a facilitare simili sviluppi: il passaggio di testimone da Walter Ulbricht ad Erich Honecker alla Segreteria del SED ebbe ad esempio una certa influenza sulle questioni di sicurezza. Honecker, già Segretario del Comitato Centrale per le questioni di sicurezza e Segretario del Consiglio Nazionale di Difesa, aveva a cuore l'argomento: venne in questo modo a costituirsi una sorta di coalizione tra apparato di sicurezza e partito, che si concretizzò in un vero e proprio asse Mielke-Honecker ${ }^{49}$. Un effetto, sebbene indiretto, ebbero anche i rivolgimenti in Cecoslovacchia. La primavera di Praga venne inserita nella solita cornice della diversione politico-ideologica: alcuni documenti della Stasi avrebbero rintracciato diversi tentativi del DGB (Deutscher Gewerkschaftsbund, il sindacato tedesco-occidentale) di sostenere, anche con aiuti finanziari, il corso riformista della politica cecoslovacca e di intensificare i rapporti tra lo stato socialista e la Repubblica Federale ${ }^{50}$. La crisi di Praga confermò in questo modo, se mai ce ne fosse stato bisogno, i timori tedesco-orientali di fronte ad un possibile avvicinamento al "nemico" 51 .

L'allargamento del Ministero, però, chiamava principalmente in causa la situazione politica emersa con la realizzazione della détente e, soprattutto, la necessità di difendersi:

Il Ministero per la Sicurezza definì come nuovi compiti il controllo e la messa in sicurezza delle nuove rappresentanze diplomatiche e dei loro lavoratori, così come la "lavorazione" di cittadini tedesco-orientali che intendevano prendere contatti con le rappresentanze occidentali; a questo andava accompagnato la sorveglianza dei giornalisti accreditati così come il controllo del traffico in aumento di visitatori e di transito dalla Germania Occidentale. [...] Lo sviluppo del personale del Ministero avvenne come espressione del bisogno di sicurezza, continuamente in aumento, da parte dei dirigenti di partito e di Stato $^{52}$.

\footnotetext{
${ }^{48}$ SÜß, Walter, op. cit., pp. 330, 331.

${ }^{49}$ GIESEKE, Jens, Die hauptamtlichen Mitarbeiter der Staatssicherheit. Personalstruktur und Lebenswelt 1950-1989, cit., pp. 293, 296-298.

${ }^{50}$ BANGE, Oliver, op. cit., p. 271.

${ }^{51}$ GIESEKE, Jens, Die hauptamtlichen Mitarbeiter der Staatssicherheit. Personalstruktur und Lebenswelt 1950-1989, cit., p. 295.

${ }^{52}$ GIESEKE, Jens, Die hauptamtlichen Mitarbeiter des Ministeriums für Staatssicherheit, Berlin, BStU, 1996, pp. 42, 43.
} 
I dati sono estremamente significativi: si calcola infatti che, complessivamente, tra la fine del 1968 e il 1982 il numero di hauptamtlichen Mitarbeiter aumentò del 147 percento, passando da 32.912 all'impressionante cifra di $81.495^{53}$. Le percentuali di aumento dei singoli dipartimenti forniscono degli importanti indicatori sulle direttive del lavoro del Ministero. La rilevanza degli intenti "difensivi” sembra ad esempio essere confermata dal notevole allargamento del Dipartimento II, deputato all'organizzazione del controspionaggio: con un passaggio da 214 a 988 unità, il personale impiegato in questa sezione aumentò del 286 percento, molto più rispetto allo stesso HVA54. Un ampliamento dovuto ad un incremento dei compiti affidati alla sezione, in conseguenza dell'apertura a Occidente: con l'ordine 16/74 il dipartimento fu incaricato della protezione del Ministero degli Affari Esteri e delle sue istituzioni nella DDR e all'estero55; con l'ordine 17/74, invece, gli fu assegnata la gestione ed il controllo di giornalisti e corrispondenti occidentali56. Un'espansione di questa portata necessitava di una sostanziale riorganizzazione, la quale comprese un significativo spostamento di competenze: per alleviare il Dipartimento II dai numerosi incarichi, la gestione del controspionaggio verso l'estero venne affidata al Dipartimento HVA. Con l'ordine 14/73 fu creata a all'interno di quest'ultimo la sezione IX, una decisione che rafforzava l'idea secondo la quale il Westarbeit sarebbe dovuto risultare da una stretta collaborazione tra spionaggio e difesa ${ }^{57}$. L'HVA stesso conobbe un'espansione significativa, sebbene non comparabile a quella del Dipartimento II: nel periodo qui considerato il personale a livello centrale aumentò, ma "solo" dell'86 percento ${ }^{5}$. Anche in questo caso, l'influsso della distensione era notevole: l'attenzione nei confronti dei pericoli, infatti, non escludeva che l'apertura verso Occidente potesse fornire una serie di occasioni utili a rafforzare lo spionaggio tedesco-orientale nella vicina Germania Federale. La Stasi aveva d'altronde già ampiamente utilizzato a proprio beneficio il cosiddetto Heimvorteil, il "vantaggio di essere di casa", il fatto cioè di avere come principale nemico un paese che utilizzava la stessa lingua e che condivideva un retroterra culturale

\footnotetext{
${ }^{53}$ GIESEKE, Jens, Die hauptamtlichen Mitarbeiter der Staatssicherheit. Personalstruktur und Lebenswelt 1950-1989, cit., p. 293.

${ }^{54}$ I dati sono calcolati considerando la sede centrale della Stasi. GIESEKE, Jens, Die hauptamtlichen Mitarbeiter des Ministeriums für Staatssicherheit, cit., p. 42.

${ }^{55}$ Per adempiere a questi nuovi obblighi venne anche istituita una nuova sezione, la numero 14. LABRENZ-WEI $\beta$, Hanna, op. cit., p. 50.

${ }_{57}^{56}$ In questo caso venne invece costituita la sezione 13. ID., p. 51

${ }^{57}$ SIEBENMORGEN, Peter, op. cit., pp. 135, 136.

${ }^{58}$ GIESEKE, Jens, Die hauptamtlichen Mitarbeiter der Staatssicherheit. Personalstruktur und Lebenswelt 1950-1989, cit., p. 317.
} 
comune59. Il transito transfrontaliero (soprattutto negli anni Cinquanta, prima della costruzione del Muro), venne ad esempio sfruttato per infiltrare propri agenti nella Repubblica Federale, che, in alcuni casi, riuscirono a raggiungere posizioni di rilievo nella vita politica ed istituzionale del paese. Il caso più noto è certamente quello di Günter Guillaume, assoldato come collaboratore dalla Stasi con nome in codice "Hansen" già dal 1955, che un anno dopo venne infiltrato in RFT: nel nuovo Paese riuscì a fare una brillante carriera nell'SPD, tanto da riuscire ad entrare nell'entourage di Willy Brandt. Il caso di spionaggio venne scoperto nel 1974, costringendo il Cancelliere alle dimissioni6o ${ }^{6 e}$ infiltrazioni documentate sono diverse e nei confronti di vari partiti, movimenti ed istituzioni tedesco-occidentali; esse non servivano solo, come si è visto, ad influenzare il corso politico, ma anche ad ottenere informazioni sul vicino occidentale. La presenza di rappresentanze diplomatiche in Germania Federale e negli altri paesi del blocco occidentale favoriva questo lavoro, permettendo il loro utilizzo come legal abgedeckten Residenturen, come centri di spionaggio coperti legalmente ${ }^{61}$. La possibilità di raccogliere notizie direttamente attraverso il Ministero per gli Affari Esteri finì col far perdere all'HVA ed alla sua rete di collaboratori non ufficiali il monopolio sui canali informativi62. Ciononostante, analogamente all'espansione del resto del Ministero, anche il numero di inoffiziellen Mitarbeiter conobbe un incremento notevole: esso superò la cifra di 200.000 tra il 1975 ed il 1977, raddoppiando nel giro di cinque anni e raggiungendo in questo periodo il suo massimo storico $^{63}$. Anche l'espansione della rete di collaboratori non ufficiali non sembrò, però, seguire una logica esclusivamente offensiva: i dati riportati da Müller-Enbergs nel suo approfondito studio sugli IM realizzato per la fondazione BStU, sembrano infatti suggerire che il numero di questi collaboratori aumentò in modo considerevole nei momenti di crisi o di potenziale difficoltà. Si può cioè registrare, ad esempio, un'espansione della rete non ufficiale in concomitanza con gli avvenimenti del 17 Giugno 1953 o a ridosso della costruzione del Muro, così come, appunto, nei primi anni della distensione inter-tedesca, proprio perché, anche in questo caso, il Ministero temeva una possibile crisi ${ }^{64}$.

\footnotetext{
${ }^{59}$ WEBER, Marije, «„Operationsgebiet BRD“. Die Bonner Republik nicht im Griff, aber im Blick der Stasi», in Akademie-Report, 4/2004, pp. 18-20.

${ }^{60} \mathrm{KNABE}$, Hubertus, Die Unterwanderte Republik, cit., pp. 44, 45.

${ }^{61}$ KNABE, Hubertus, «Die Stasi als Problem des Westens. Zur Tätigkeit des MfS im „Operationsgebiet“», cit., p. 9.

${ }_{62}^{62}$ MÜLLER-ENBERGS, Helmut, Die inoffiziellen Mitarbeiter, cit., p. 15.

${ }^{63}$ Cfr. Ibidem, tab. 9, p. 36-37.

${ }^{64}$ Ibidem, p. 37.
} 
La storiografia tedesca sembra così suggerire che la distensione sviluppatasi in Europa nel corso degli anni Settanta provocò una reazione di tipo prevalentemente difensivo nel Ministero per la Sicurezza Statale della DDR. Ciò non toglie che la condizione derivatane abbia favorito una parallela attività offensiva, concretizzatasi con l'infiltrazione in partiti politici, movimenti (da ultimo quello pacifista), istituzioni ed organizzazioni di varia natura. Gli storici tedeschi, però, sul tema sembrano dividersi: se, ad esempio, c'è chi si riferisce (come Hubertus Knabe) alla Germania Occidentale come ad una vera e propria «Repubblica infiltrata» («unterwanderte Republik»), altre voci sostengono che le infiltrazioni - che certamente vi furono - non abbiano determinato (e nemmeno influenzato in maniera risolutiva) il processo politicodecisionale tedesco-occidentale ${ }^{65}$. Le "manovre attive" condotte nel limitato periodo della distensione furono prevalentemente tese alla raccolta di informazioni ed al sostegno di quelle forze progressiste, nello specifico l'SPD, più orientate ad una politica di avvicinamento verso la DDR. Tale attività, però, sembra aver dato seguito alle esigenze politiche del partito: se infatti, da una parte, il superamento della dottrina Hallstein ed il conseguente ottenimento del riconoscimento internazionale erano obiettivi vitali della politica estera della DDR di quegli anni, dall'altra le analisi tedesche consegnano l'immagine di un Ministero molto preoccupato dai pericoli e dalle insidie che da quel processo sarebbero scaturite. Complice di una simile percezione fu, certamente, la filosofia di sicurezza già ampiamente radicata nella Stasi: il concetto di diversione politico-ideologica, così come la concezione di un Westarbeit costituito da una commistione di spionaggio e difesa, infatti, sembrerebbero confermare quella che Tobias Wunschik ha definito «ipocondria della sicurezza politica» ${ }^{66}$. In linea generale, sia in senso difensivo che offensivo, la risposta del Ministero alla distensione si concretizzò in una straordinaria espansione dei suoi dipartimenti e delle sue attività, che è rimasta un'eccezione rispetto a qualsiasi altra fase della (breve) storia tedescoorientale.

\footnotetext{
${ }^{65}$ MÜLLER-ENBERGS, Helmut, Die Erforschung der Westarbeit des MfS - Stand und Perspektiven, cit., pp. 251, 252.

${ }^{66}$ WUNSCHIK, Tobias, «I servizi segreti e il terrorismo di sinistra della Repubblica federale e nella Ddr», in Ricerche di Storia Politica, XI, 3/2008, pp. 311-326, p. 325.
} 


\section{${ }^{*}$ L'autore}

Federica Addis è laureata in Scienze Politiche presso l'Università degli Studi di Padova. È dottoranda borsista in Storia dell'Europa dal Medioevo all'Età Contemporanea presso l'Università degli Studi di Teramo. Il suo progetto di ricerca dottorale mira ad indagare l'atteggiamento della Repubblica Democratica Tedesca nei confronti della sinistra extraparlamentare, della violenza politica e del fenomeno terroristico in Italia tra il 1968 e la fine degli Anni Ottanta.

URL: < http://www.studistorici.com/progett/autori/\#Addis >

\section{Per citare questo articolo:}

ADDIS, Federica, «Tra spionaggio e difesa: il Westarbeit tedesco-orientale nella fase di détente», Diacronie. Studi di Storia Contemporanea : La voce del silenzio: intelligence, spionaggio e conflitto nel XX secolo, 29/12/2016, URL:< http://www.studistorici.com/2016/12/29/addis_numero_28/ >

\section{Diacronie Studi di Storia Contemporanea $\beta$ www.diacronie.it}

Risorsa digitale indipendente a carattere storiografico. Uscita trimestrale. redazione.diacronie@hotmail.it

Comitato di redazione: Jacopo Bassi - Luca Bufarale - Antonio César Moreno Cantano - Deborah Paci - Fausto Pietrancosta - Alessandro Salvador - Matteo Tomasoni - Luca Zuccolo

Diritti: gli articoli di Diacronie. Studi di Storia Contemporanea sono pubblicati sotto licenza Creative Commons 3.0. Possono essere riprodotti e modificati a patto di indicare eventuali modifiche dei contenuti, di riconoscere la paternità dell'opera e di condividerla allo stesso modo. La citazione di estratti è comunque sempre autorizzata, nei limiti previsti dalla legge. 